\title{
A Code Based Approach to Image-Processing Teaching
}

\author{
A. Biancardi and M. Pini \\ Università di Pavia \\ Via Abbiategrasso 209 - 27100 Pavia - Italy \\ e-mail: albertodipvvis.unipv.it \\ maxi@ipvis.unipv.it
}

\begin{abstract}
The Pacco Teaching Tools library extends Pacco, an object-oriented data-processing environment, and offers a new approach to i.p. teaching where the source code is placed at the centre of the educational experience, providing a common framework between theory and practice and accomplishing students' learning of the development environment while improving their knowledge of i.p. techniques. Thanks to the automatic monitoring of processed objects and to the interactivity and robustness of the tools, the environment seems very suited to the targeted task.
\end{abstract}

\section{Introduction}

The increasing diffusion of image processing (i.p.) courses at the (uder)graduate level makes even more glaring the almost total absence of tools to support and complement i.p. teaching.

The accepted evolution of a course can be roughly divided into four main stages, going in students' increasing involvement:

1 exposition of ip techniques and strategies

2 learning of the development system

3 experimental verification of theoretical results

4 project implementation

The big problem with i.p. teaching is not really the fact that examples and results require images and graphical items and that they cannot be easily described by means of words; the difficulty lies in the unavoidable dynamicity of those images that complement a lecture. Moreover it is easier to keep user's attention focused on the topic if a set of evolving pictures is presented (e.g. choosing a data file, performing an image transform or a special task, showing effects and results, choosing another file, and so on). This way theory becomes more interesting and not boring.

The almost interactive nature of this process means that some kind of active-textbook should be used. If this guideline is coupled with the importance of teaching not only the theoretical techniques but also the way to perform them, it follows that the programming development environment is the best candidate to act both as an activetextbook and as a workbench.

Anyway the limited time students have to get aquainted to the development environment plays a major role in the academic context. The ideal environment should: 
- offer a smooth learning curve

- get students productive as soon as possible

- do not encumber them with eccessive details

Finally, another issue worth considering is the ability to provide autonomous or self-paced learning, which is strictly connected to the management of on-line (and off-line) documentation together with some kind of help facility.

To cope with these requirements, the Pacco Teaching Tools (PTT) library was developed to let Pacco, an object-oriented data-processing environment[1], become even more effective as an authoring system and a development environment for non-experienced programmers.

Before peeking into the details of the PTT library, a brief overview of Pacco will be given. Features, use and implementation of PTT will follow, while the discussion of related work will conclude the paper.

\section{Compound Objects and Active Components}

Pacco stands for "Pavia Active-Component Compound Objects" and is an extension of a general purpose command language (Tcl) and its graphical toolkit (Tk)[2]. Pacco design is characterized by a new approach to object-oriented programming where run-time extensions, coded in $\mathrm{C}$ language or Tcl, are used to enhance the behaviour of objects and to supply unique services, such as transparent feedback to data changes.

In order to allow the programmer capture data dependent structures and relationships, Pacco defines a new object model by introducing the concept of compound object, which is called box, of container class, named Cbox as child-box, and of unknown class, while reserving the usual concept of object [3] to standard classes.

Pacco objects are tree structures made up of components; each component belongs to a class and Cbox components are both embedded (being components) and embedding (like boxes), thus allowing the creation of the tree hierarchy.

Boxes are the atomic items as far as object creation and destruction is concerned, while components are the atomic information holders as far as the tcl API is concerned; finer grained data inside a component can only be accessed by means of class functions, named actions and written in C-language.

The clear-cut division between structuring and information processing, which is enforced by the box/component model, allows Pacco to handle most of the object-management chores disregarding completely the data-types of the components being managed. Therefore whichever task can be performed in a type-independent way is assigned to the kernel. It deals with computation dispatching and result gathering. It is in charge of data input/output handling at the box or Cbox level. It performs loading of unknown classes and actions on demand. It centrally manages the retrieval of defaults for any Pacco feature that can be given a default value.

The most peculiar kernel service is the bind action. It allows the Tcl programmer to add a list of commands to be executed whenever the data of a component change (or the box is destroyed when the command is bound to the whole box). This capability allows the easy implementation of data-driven applications. Moreover, the ability to freely intermix data-generated and user-generated events opens the way to an effective realization of reactive scripts. 
Pacco defines a new tcl command named after the box anytime a new box is created. Each component in the box gets a tcl command, too. These commands are used to invoke actions to be performed over the component or box. This is whence active component comes from. Component command act as component identifiers as well and may be used to uniquely reference components.

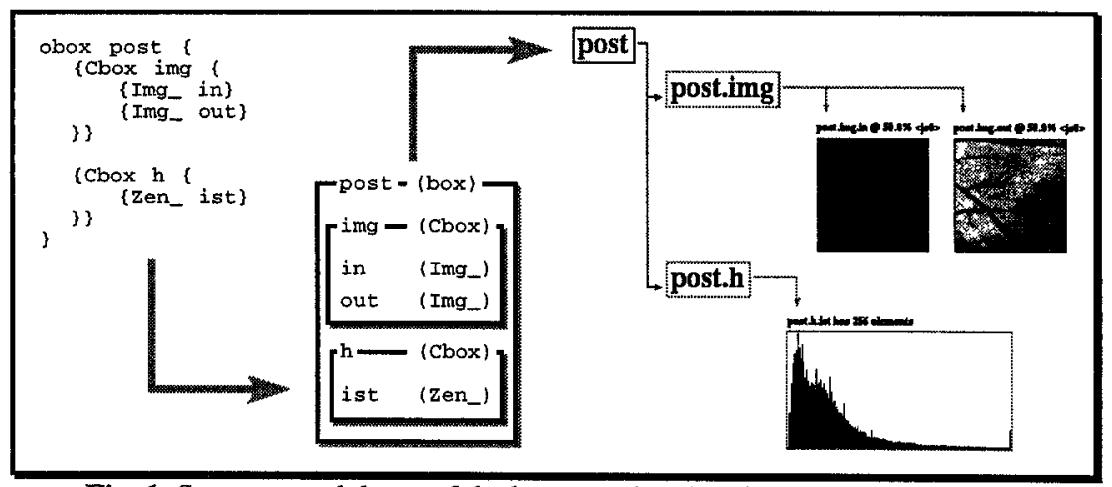

Fig. 1. Structure and dump of the box associated to the command example.

graphic library extends Tk, the tcl-based X11[4] toolkit, with a set of new widgets and canvas items that can be linked directly to Pacco components. A number of utility procedures further enhance the environment with a polymorphic display command, region of interest handling, colormap animation support and so on. Bindings show their usefulness by allowing the automatic update of component visualization.

Pacco is available by anonymous ftp at iride.unipv.it in the directory /pub/Pacco.

\section{Pacco Teaching Tools}

Being a command language, $\mathrm{tcl}$ is simple, has a minimal syntax and is similar to commonly used unix-shells; therefore learning tcl is almost immediate (at least as far as basic concepts and command set are concerned).

Moreover the powerful combination of tcl, tk and Pacco creates a functionally rich environment making straightforward the implementation of interactive tools that keep track of data changes.

Thus, box visualization and monitoring can be supplied transparently through the redefinition of the box-creating command. (Fig. 1 and 2 show two dumps of such windows into directly created PostScript[5] files)

The coesistence of this two factors opens the way to a new approach to i.p. teaching which is embodied by the PTT library. The idea is that the code, implementing an i.p. task, can act both as a reference of the task and as the way to perform the task. In short, it makes possible to provide a common framework between theory and practice and to accomplish students' learning of the development environment while improving their knowledge of i.p. techniques. 
In addition to the monitoring support, the library deals with the authoring system and a special command tool to ease code creation and development.

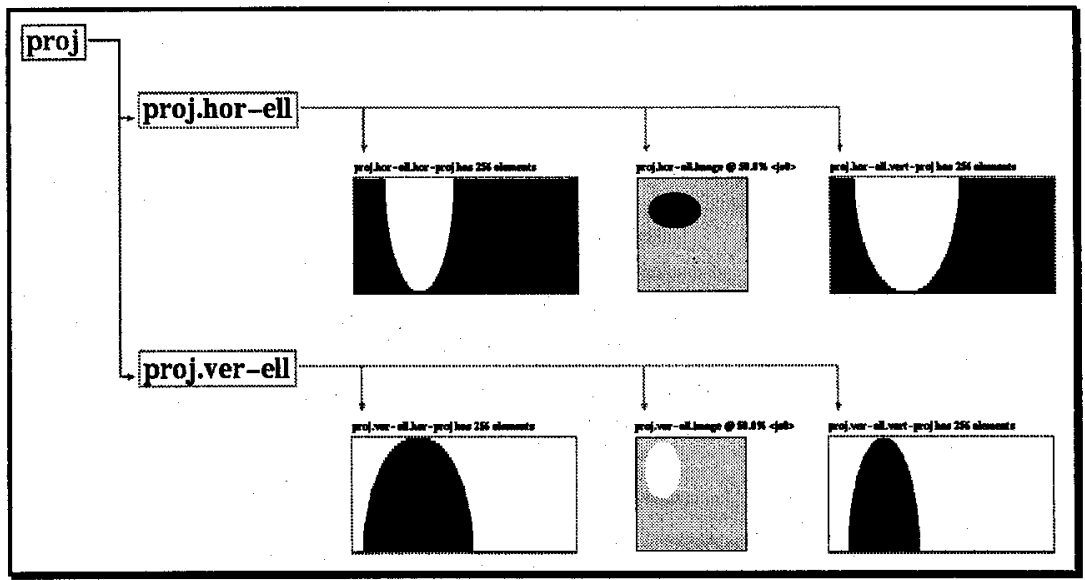

Fig. 2. Dump of the monitoring window associated to the tutorial example.

\section{"Hands On" Tutorials}

Each PTT tutorials is structured into sections, one for each specific sub-topic. Every section has the following common structure: a global description of its goals and algorithm, the section source code, subdivided into groups of instructions called steps, and a set of captions, which are synchronized to and explain the code steps.

Thanks to the code-based approach, the authoring tool (Fig. 3) is a specially tailored tcl-source debugger. Navigation controls are the natural translation of the debugger execution commands into the new context, extended by a kind of undo feature where the user can restart the current step. Synchronization is achieved by tagging the code according to the step it is related to, thus allowing the user to edit the code without disrupting existing links.

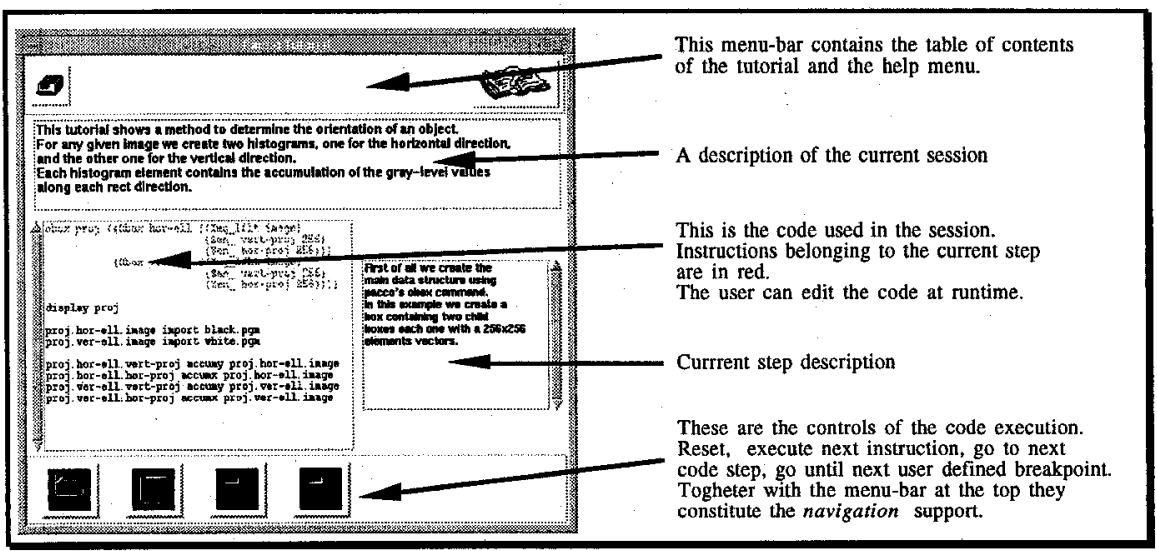

Fig. 3. The tutorial window. 
The resulting environment is highly interactive and robust; it should attract users' exploration and therefore be very suited to novices, as experience proved [6].

One problem, currently being addressed, is the implementation of a tool to speed up tutorial creation and caption translation into another language (Pacco structured objects already offer an ideal way to store tutorials).

\section{Project Implementation Support}

The command tool (Fig. 4) is intended as a first attempt at giving a better visibility and history handling to pacco programmers. Just like visual programming systems, the command tool tries to expose all the available commands, to make it easier to track a working-session evolution and to retrieve past commands or command results. For example if the first item of the command line is a pacco's component, pressing a mouse button in the command line builds up a pop-up menu showing all the actions available for that component; selecting an action pastes its name into the command line.

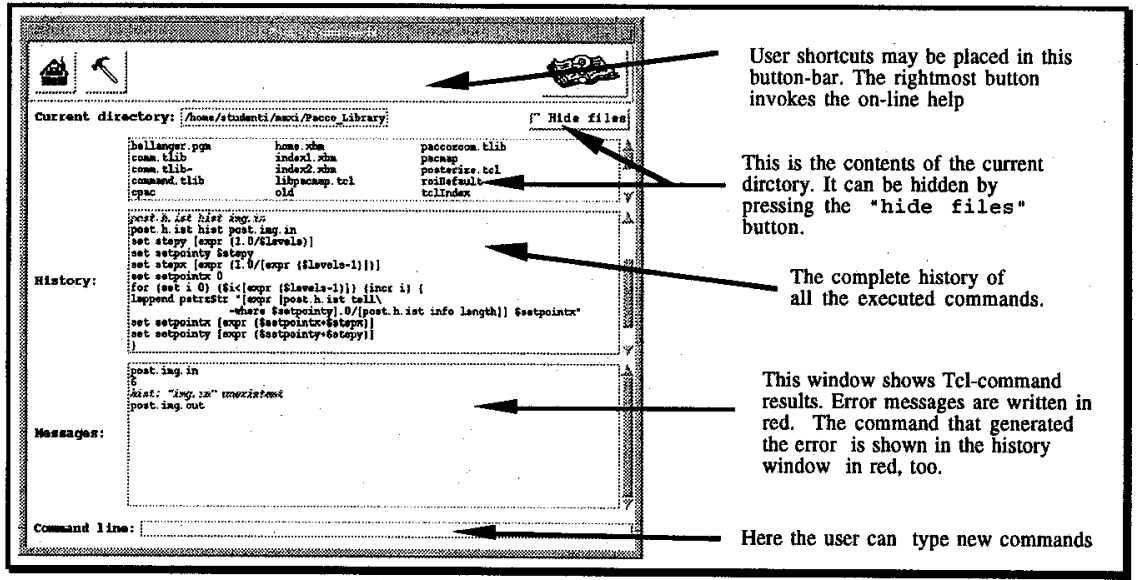

Fig. 4. The command window.

\section{Related Work}

Many packages try to be simple and accessible (like Vista[7] or LaboImage[8]) so that they can be used in research and didactic environments. Anyway, up to now, only Khoros [9] explicitly addressed the problem.

The DIP Toolbox[10] of Khoros 2.0 relies on a www-client and cantata, Khoros visual programming (VP) tool. The author's main claim states that visual programming is the only means to effectively hide the huge complexity of the system.

While a deeper discussion on merits and pitfalls of VP is beyond the scope of this paper, a few comments may be sketched out:

- if students already know how to program and are used to a unix shell, extending their programming skills with a very-high-level command language is not an issue, at all;

- flow-control is sometimes difficult to express in a VP tool [11]; 
- the density of information in a VP layout cannot be higher than in a textual program;

- it is possible to supply a VP layer to tcl-based environments [12].

\section{Conclusions}

The PTT library offers a new point of view to i.p. teaching by placing the source code at the centre of the educational experience. Thanks to the automatic monitoring of processed objects and to the interactivity and robustness of the tools, the environment seems very suited to the targeted task.

\section{References}

1. A. Biancardi and A. Rubini, Pacco - An Effective Approach To I.P. Programming, Proc. 12th ICPR, IEEE Press, Los Alamos, 1994

2. J.K. Ousterhout,Tcl and the Tk Toolkit, Addison-Wesley, Reading, 1994.

3. L. Cardelli and P. Wegner, On Understanding Types, Data Abstraction, and Polymorphism, Computer Surveys, Vol. 17, Num. 4, 1985.

4. R. Schifler and J. Gettys, X Window System, Digital Press, 1990.

5. Adobe System Incorporated, PostScript Language Reference Manual, AddisonWesley, Reading, 1985.

6. J. M. Carroll, Minimalist Design for Active Users, in B. Shackel(ed.), Human Computer Interaction - Interact'84, North-Holland, Amsterdam, 1985, pp 39-44.

7. A. Pope and D. Lowe, Vista: A Software Environment for Computer Vision Research, Proc. of Intl. Conf on Computer Vision and Patter Recognition, 1994.

8. A. Jacot-Descombes M. Rupp and T. Pun, LaboImage: a portable window-based environment for research in image processing and analysis, 1992 SPIE/IS\&T Symposium on Electronic Imaging, SPIE Proceedings Vol. 1659, 1992.

9. J. Rasure and S. Kubica, The Khoros Application Development Environment, in H.I Christensen and J.L Crowley (ed.), Experimental Environments for Computer Vision and Image Processing, World Scientific 1994.

10. R. Jordán and R. Lotufo, Digital Image Processing with Khoros 2.0, available at the URL http://www.khoros.unm.edu/dip/html/dip.html (and following pages).

11. C. Balasubramanian, Dataflow image processing, Computer, November 1994, pp 82-84.

12. M. Bernini and M. Mosconi, VIPERS: a Dataflow Visual Programming Environment Based on the Tcl Language, Proc. of the Workshop on Advanced Visual Interfaces AVI'94, ACM Press, 1994. 